\title{
Probability in the Grammar of German and Dutch: Interfixation in Triconstituent Compounds*
}

\section{Andrea Krott ${ }^{1}$, Gary Libben², Gonia Jarema ${ }^{3}$, Wolfgang Dressler ${ }^{4}$, Robert Schreuder $^{5}$, Harald Baayen ${ }^{5}$}

${ }^{1}$ University of Birmingham, U.K.

${ }^{2}$ University of Edmonton, Canada,

${ }^{3}$ University of Montreal, Canada

${ }^{4}$ University of Vienna, Austria

${ }^{5}$ University of Nijmegen, The Netherlands

\section{Key words}

compound structure

distributional properties

interfixes

multiconstituent compounds

separation hypothesis

\begin{abstract}
This study addresses the possibility that interfixes in multiconstituent nominal compounds in German and Dutch are functional as markers of immediate constituent structure. We report a lexical statistical survey of interfixation in the lexicons of German and Dutch which shows that all interfixes of German and one interfix of Dutch are significantly more likely to appear at the major constituent boundary than expected under chance conditions. A series of experiments provides evidence that speakers of German and Dutch are sensitive to the probabilistic cues to constituent structure provided by the interfixes. Thus, our data provide evidence that probability is part and parcel of grammatical competence.
\end{abstract}

\section{Introduction}

Multiconstituent compounds such as German Schachweltmeistertitel ('chess world champion title', i.e., world chess championship) pose a special challenge to theories of lexical access. While a clause such as the title of being the world's champion in chess provides a great many syntactic cues guiding interpretation, no such cues are available for the

* Acknowledgments: The authors are indebted to Arne Fitschen and Ulrich Heid (University of Stuttgart) for providing a list of 34,000 German compounds. This study was made possible by an SSHRC MCRI grant to G. Libben, G. Jarema, E. Kehayia, B. Derwing, and L. Buchanan, and was also supported by a PIONIER grant of the Dutch Research Council NWO to H. Baayen.

Address for correspondence. Andrea Krott, School of Psychology, University of Birmingham, Edgbaston, Birmingham B15 2TT, United Kingdom.

Phone+44(0)121 4144903, e-mail<a.krott@bham.ac.uk>. 
interpretation of Schachweltmeistertitel. Nevertheless, speakers of German understand this recursively structured compound just as well as its phrasal paraphrase, assigning it a dependency structure such as [[ chess [world champion]]title], in which Meister is the head of Weltmeister, Weltmeister is in turn the head of Schachweltmeister, and Titel the head of the full compound. Note that this compound as a whole has a left-branching structure, but that its left constituent itself has a right-branching structure. Note, furthermore, that the principles of recursivity and right-headedness ${ }^{1}$ by themselves do not constrain the interpretation of this compound. Structurally, Schachweltmeistertitel might equally well be parsed into Schachwelt and Meistertitel. The issue addressed in this study is which principles (in addition to recursivity and right-headedness) might guide readers and listeners when interpreting German and Dutch multiconstituent compounds such as Schachweltmeistertitel which consist of a sequence of monomorphemic nouns. ${ }^{2}$

Our leading hypothesis is that comprehension of such compounds is guided by the statistical distributional properties of the existing compounds in the mental lexicon, even when the differences in the distributions are subtle and subject to substantial variation. One such distributional property might be the distribution of left-branching and right-branching compounds. If the majority of compounds in the mental lexicon is left-branching, this might bias comprehension towards expecting existing and novel multiconstituent compounds to be left-branching as well.

Another type of distributional information that might be crucial for comprehension is the availability of smaller compounds in the mental lexicon that might serve as larger building blocks. Instead of trying to construct an interpretation for Fussballweltmeistertitel from the individual simplex nouns Fuss, Ball, Welt, Meister, and Titel, one might in fact need only construct an interpretation for the sequence of Fussball, Weltmeister, and Titel. The greater the frequency of an embedded compound, the more likely it might be to serve as a pre-existing unit for interpretation.

If a language has no interfixation (as in English or French), or if interfixation is phonologically conditioned and obligatory (as in Polish or Greek), then the distribution of branching direction and the information provided by existing compounds embedded as constituents is the only linguistic information available for guiding interpretation. However, the interfixes that are found in roughly a third of German and Dutch compounds might provide an additional distributional source of information. In these languages, the interfixes, also referred to in the literature as linking elements or linking morphemes, can be traced to older case and number endings. In Dutch, they are, with a few exceptions, all that is left of the rich inflectional system that characterized medieval Dutch. In German, the interfixes are homophonous with present-day case and number endings, but their distributional properties, as will be described in more detail below, differ from those of genuine inflectional formatives.

1 German and Dutch compounds follow William's (1981) right hand head rule, thus recursive compounding is always head-final. See also Haider (2001) for German, and Booij (2002) for Dutch.

2 The additional guidance provided by verb argument structure in the case of compounds with deverbal constituents such as dishwasher falls outside the scope of this study. 


\section{TABLE 1}

Examples of left-branching and right-branching triconstituent compounds in Dutch with the interfix $-s-$

scheep-s-bouw+maatschappij

s left

left-branching

ship building company

arbeid-s-vraag+stuk

s left

right-branching

employment question-issue

krijg-s-man-s-eer

s left and right

left-branching

war man honor

rijk-s-arbeid-s-bureau

s left and right

right-branching

national employment office

grond+wet-s-artikel

s right

left-branching

ground law article, constitution

hoofd+verkeer-s-weg

s right

right-branching

main traffic road

Table 1 provides some examples of Dutch triconstituent compounds with the interfix $-s-$. Note that this interfix may occur between the first and the second constituents, between the second and the third constituents, or even at both these positions. In addition, a compound can be right-branching or left-branching. If the distribution of the -s- were as uniform as Table 1 would have us believe, then the $-s$ would not be of any use for constructing the interpretation of multiconstituent compounds. However, Grimm (1878) and in his wake, Žepić (1970), Fleischer (1976), and Fuhrhop (1998; 2000), among others, have pointed out that this is not the case. In many left-branching compounds, the interfix occurs in the second interfixation slot between the two immediate constituents of the compound. These scholars suggest that it is the function of the $-s$ - to separate the compound into its immediate constituents. We will refer to this possibility as the separation hypothesis.

The present study first presents a lexical statistical survey of the distribution of the interfixes of Dutch and German as a function of their position in left and right branching compounds. The goal of this survey is to establish to what extent the $-s-$ interfix and the other interfixes of German and Dutch indeed reveal a preference for the main constituent boundary. We will call this aspect of the separation hypothesis the distributional separation hypothesis. In addition, this study presents a series of experiments that seek to clarify whether speakers of German and Dutch indeed make use of interfixes to determine the main constituent boundary in compounds. We will call this aspect of the separation hypothesis the functional separation hypothesis. Before addressing the distributional and functional separation hypotheses, however, we first provide additional details of the distributional properties of the interfixes in German and Dutch. 


\section{Interfixes in German and Dutch}

The German interfixes -s-, - (e)n-, -es-, -e-, -er-, -ens- and the Dutch interfixes -s-, $-e(n)-,-e r-$ are found in roughly a third of nominal compounds. Historically, interfixes are case and number endings of left constituents. In Dutch, the -e- may also have formed part of the stem before schwa-apocope applied (Booij, 2002). In some compounds, one can still interpret the interfix as a genitive or plural marker (e.g., German Kapitän $+s+$ kajüte 'captain + GENITIVE + cabin'; Dutch abrikoz + en + taart 'apricot + PLURAL + tart'). However, although homophonous with modern case and number endings (in German) and with plural markers (in Dutch), the interfixes do not have the same distributional properties. For instance, the German noun Liebe 'love' cannot be combined with the case marker $-s$, nor with the plural marker $-s$, but it does occur with the interfix $-s$ - in Liebe $+s+$ brief 'love + INTERFIX + letter'. The same is true for the Dutch noun schaap 'sheep' that occurs with $-s$ - in schaap $+s+$ leder 'sheep + INTERFIX + leather' but that occurs with -en- in the plural schaap-en. This is not to say that interfixes are by definition meaningless. To the contrary, Schreuder, Neijt, Van der Weide, and Baayen (1998) have shown that in Dutch the interfix -eninduces a plural interpretation for the left constituent.

An indication that the interfixes are not mere relics of the past, as in the case of the English interfix $-s$ - in helmsman, is that interfixes are used productively in novel compounds. In fact, the distribution of interfixes in novel and existing compounds has been one of the enigmas of German and Dutch morphology, as there are no hard and fast syntagmatic rules governing their use, especially when the left constituent is a monomorphemic noun.

Dressler, Libben, Stark, Pons, and Jarema (2001) have argued for German that the choice of the interfix is sensitive to inflectional microclasses. Krott, Baayen, and Schreuder (2001) have argued for Dutch, and Krott, Schreuder, Baayen, and Dressler (in Krott, 2001, see also Libben, Jarema, Dressler, Stark, \& Pons, 2002) have similarly argued for German, that an even more tightly constrained paradigmatic systematicity is at issue. They show that the distribution of interfixes in the sets of compounds sharing the left constituent (and also to some extent those sharing the right constituent) is predictive of the choice of the interfix in novel compounds and of the time required to make this choice. One of the questions to be considered in the present study is to what extent the paradigmatic systematicity governing the distribution of interfixes leaves room for the separation hypothesis as an additional principle shaping this distribution.

Another important factor governing the choice of the interfix is whether the left constituent has a derivational suffix as head. If so, this suffix may govern the selection of the interfix. This is the only circumstance in which some clear exceptionless syntagmatic rules are operative. In Dutch, for instance, all compounds that have a diminutive as left constituent contain the -s-interfix. Aronoff and Fuhrhop (2002) have pointed out for German that especially suffixes that do not allow further word formation, that is, that have a morphological valency of zero, are most likely to require an interfix when embedded as a left constituent in a compound. They suggest that for such compounds the interfix has the function of opening the left constituent for further word formation. Krott, Schreuder, and Baayen (2002) provide distributional evidence that Dutch interfixes may serve a similar opening function. Note that 
wherever the choice of the interfix is grammaticalized deterministically, as in the case of Dutch diminutive left constituents, the question of the additional explanatory power of the separation hypothesis again arises.

In what follows, we first present the results of our survey of the lexical statistics of interfixation in German and Dutch. This survey is restricted to compounds consisting of simple nouns. By excluding all compounds from the analysis in which the presence of the interfix is determined by other factors, such as derivational suffixes on nonfinal constituents, the relation between the hierarchical organization of the compound and the distribution of interfixes can be established most clearly. For a brief discussion of interfixation in triconstituent compounds with derived constituents, the reader is referred to the Appendix.

Following the lexical statistical survey, we present a series of experiments investigating whether and how the interfixes affect the way in which speakers of German and Dutch interpret the structure of multiconstituent compounds. All experimental studies of compound processing that we are aware of have addressed the production or comprehension of compounds consisting of two constituents. For such compounds, the question of their branching direction does not arise. The experiments presented here address, for the first time, the assignment of structure to compounds with three constituents. The goal of these experiments is to establish the extent to which the distributional properties of existing compounds in the lexicon, and specifically the distribution of interfixes within these compounds, guide people's interpretations.

\section{Lexical statistics}

Table 2 lists, for German and Dutch, the number of compounds with two, three, and four simple nominal constituents (henceforth simple nominal compounds), as well as the proportions of compounds without interfixes. The counts for German are based on newspaper corpora comprising some 76 million words, as well as on the German data in the CELEX lexical database (Baayen, Piepenbrock, \& Gulikers, 1995), which are based on a corpus of some six million words. The counts for Dutch are based on the Dutch section of the CELEX lexical database, which is based on a corpus of approximately 42 million words.

\section{TABLE 2}

Compounds in German and Dutch consisting of simplex nouns and optionally interfixes

\begin{tabular}{lcrc}
\hline language & $\begin{array}{c}\text { number of } \\
\text { constituents }\end{array}$ & $\begin{array}{c}\text { number of } \\
\text { compounds }\end{array}$ & $\begin{array}{c}\text { proportion without } \\
\text { interfixes }\end{array}$ \\
\hline German & 2 & 6643 & 0.65 \\
& 3 & 442 & 0.57 \\
\hline \multirow{2}{*}{ Dutch } & 4 & 5 & 0.80 \\
& 2 & 14644 & 0.73 \\
& 3 & 546 & 0.67 \\
& 4 & 6 & 0.67
\end{tabular}


First note that the number of compounds with two constituents is much larger than the number of compounds with three constituents, and that the number of compounds with four constituents is extremely small, both in German and in Dutch.

Surprisingly, the number of two-constituent compounds in German is less than half the corresponding number in Dutch (6643 vs. 14644). This suggests that compounding might be more productive in Dutch than in German. This possibility receives further support from the total counts of noun-noun compounds (including derived constituents) in our data sets: 44,000 for Dutch versus 32,000 for German, in spite of the fact that the German data are based on corpora that together are twice as large as the Dutch corpus. It should be kept in mind, however, that the Dutch corpus samples a much broader range of registers. Moreover, comparative studies of morphological acquisition studies suggest that compounds emerge more rapidly with German speaking children than with children learning Dutch (see, e.g., Dressler, Kilani-Schoch, \& Klampfer, in press). We leave this issue to further research.

Focusing on the compounds with two and three constituents, we find that the proportion of two-constituent compounds with no interfix is larger than the corresponding proportion for three-constituent compounds, both in $\operatorname{German}\left(\chi^{2}(1)=11.19\right.$, $p=.0008$, here and elsewhere, all chi-squared tests are run with continuity correction), and in Dutch $\left(\chi^{2}(1)=7.36, p=.0067\right)$. In other words, three-constituent compounds are more likely to contain interfixes (one, possibly even two) than two-constituent compounds. This observation is in line with what Žepić (1970) and Fuhrhop (1998) report for German.

This increase in the use of interfixes in triconstituent compounds constitutes a first potential piece of evidence in favor of the separation hypothesis, namely that in triconstituent compounds interfixes have the function of marking the major constituent boundary. However, in order to evaluate this potential evidence, we first have to consider other possible factors that might lead to such an increase. For instance, this increase might be due simply to there being two positions available for interfixation instead of just one. In fact, the 442 German triconstituent compounds provide 884 slots for interfixation, of which 232 are used, which amounts to $26.2 \%$ (There are 190 compounds with 1 interfix and 21 compounds with 2 interfixes.) This is a significantly reduced realization rate compared to that of the biconstituent compounds $\left(35.0 \%, \chi^{2}(1)\right.$ $=26.3 ; p<.0001)$. Similarly, the 546 triconstituent Dutch compounds provide 1092 slots for interfixation, of which only 214 are actually used: $18.0 \%$ (There are 180 compounds with 1 interfix and 17 compounds with 2 interfixes). Compared to the biconstituent compounds, for which $27 \%$ of the positions are realized, we again have a significant reduction $\left(\chi^{2}(1)=28.2 ; p<.0001\right)$. Thus it is not the case that the increase in the number of interfixes in triconstituent compounds is simply the result of the doubling of the available slots.

Interestingly, there are surprisingly few compounds with two interfixes: 21 for German and 17 for Dutch. The realization rates of interfixes in biconstituent compounds are 0.35 for German and 0.27 for Dutch (see Table 2, which lists the complementary nonrealization rates 0.65 and 0.73 respectively in the last column). Binomial tests suggest that the probabilities of having 21 or fewer compounds with two interfixes in German and of having at most 17 such compounds in Dutch are vanishingly small $(p<.00001)$. This shows that the increased proportions of interfixation for the 
triconstituent compounds compared to the biconstituent compounds are also not due to the existence of large numbers of compounds with two interfixes. In fact, the opposite is true: There are far fewer such compounds than one would expect under chance conditions. We conclude that the overrepresentation of interfixes in triconstituent compounds compared to biconstituent compounds is a nontrivial fact that requires further explanation. The separation hypothesis provides one such explanation. To evaluate this hypothesis, we must take into account whether a triconstituent compound is left branching or right-branching, as the direction of branching determines the position of the major constituent boundary. We therefore begin by surveying the distributional properties of left and right-branching compounds in German and Dutch in our databases, which are summarized in Table 3. (Note that due to the different sizes of the corpora for German and Dutch, the numbers of types and their token frequencies cannot be directly compared across languages.)

\section{TABLE 3}

Type frequency for left and right branching compounds with three simplex nominal stems in Dutch and German. Types: number of such compounds; C12: number of compounds for which the first two nouns form an existing compound; median C12: their median frequency; C23: number of compounds for which the second and third noun form an existing compound; median C23: their median frequency

\begin{tabular}{lllrrrr}
\hline language & branching & types & C12 & $\begin{array}{c}\text { median } \\
\text { C12 }\end{array}$ & C23 & $\begin{array}{c}\text { median } \\
\text { C23 }\end{array}$ \\
\hline German & left & 287 & 287 & 62 & 67 & 44 \\
German & right & 155 & 22 & 21 & 155 & 126 \\
Dutch & left & 347 & 341 & 190 & 25 & 20 \\
Dutch & right & 199 & 20 & 25 & 193 & 365 \\
\hline
\end{tabular}

The first thing to note is that there are more left-branching compounds than rightbranching compounds, both in German and in Dutch. Ortner and Müller-Bollhagen (1991) also report a preference for left-branching compounds in German. This suggests that left branchingness is the unmarked structure for a triconstituent compound.

The fourth column of Table 3, labeled ' $\mathrm{C} 12$ ', lists the number of triconstituent compounds for which the first two nouns form a compound (henceforth a C12 compound) that is also attested in our databases. In German, all 287 left-branching compounds have a C12 compound matching the left branch. In Dutch, 341 of the 347 compounds similarly consist of a C12 compound followed by another noun.

A similar pattern emerges for right-branching compounds. In German, all 155 right-branching compounds have a right branch that is an attested C23 compound. For Dutch, 193 of the 199 right-branching compounds have an attested right-branch C23 compound.

Occasionally, existing compounds straddle the major constituent boundary. In our German database, there are 67 left-branching compounds for which the C23 compound exists. A German example of such a compound is $($ Auto + Bahn $)+$ Polizei, 
'highway police', with "Bahn + Polizei," 'railway police', straddling the main constituent boundary. The median frequency (44) of these spurious compounds, however, is lower than the median frequency (62) of the correct $\mathrm{C} 12$ compounds. This difference, however, is not significant ( $W=9849.5 ; p=.408$, Wilcoxon test). The reverse pattern holds for the right-branching compounds, for which the correct C23 compounds have a higher median frequency (126) than the spurious C12 compounds (median frequency 22), a difference which is significant ( $W=883 ; p=0.0008)$. An example of such a compound is Aufklärung $+s+($ Flug + Zeug), 'spy plane', where Aufklärungsflug, 'spy flight', straddles the main constituent boundary. Table 3 shows exactly the same pattern for Dutch, with more pronounced differences among the median frequencies, both of which are significant (left branching: $W=6827$, right branching: $W=534.5$, $p<.0001$ in both cases). What these distributional data show is that there is a strong bidirectional implicational relation between frequency and left versus right branching. If the $\mathrm{C} 12$ but not the $\mathrm{C} 23$ compound exists, the triconstituent compound is almost always left-branching. If only C23 exists, the triconstituent compound is almost certainly a right-branching compound. Conversely, if a compound is left-branching, we can infer that the $\mathrm{C} 12$ exists. Similarly, if a triconstituent compound is rightbranching, the $\mathrm{C} 23$ compound is extremely likely to exist.

The relation between the frequency of the embedded compound and its position in the triconstituent compound raises the question of what the added functionality of interfixes as separators of the immediate constituents might be. After all, nearly all our triconstituent compounds are composed of an existing compound and a monomorphemic noun.

With this correlation of frequency and immediate constituent structure in mind, we now consider whether interfixes indeed show a strong preference for the major constituent boundary, as predicted by the separation hypothesis.

\section{TABLE 4}

Number of interfixes realized after the first noun only (Slot 1 only), after the second noun only (Slot 2 only), or after both the first and the second noun (Slot 1 and Slot 2) for German and Dutch left and right branching compounds with three simplex noun constituents. The numbers of compounds with the interfix $-s$ - are listed separately

\begin{tabular}{|c|c|c|c|c|c|c|c|c|}
\hline \multirow[t]{2}{*}{ language } & \multirow[t]{2}{*}{ branching } & \multirow[t]{2}{*}{ types } & \multicolumn{2}{|c|}{ Slot 1 only } & \multicolumn{2}{|c|}{ Slot 2 only } & \multicolumn{2}{|c|}{ Slot 1 and Slot 2} \\
\hline & & & all interf & es $s$ & all interf & $s$ & all interf & $s$ \\
\hline German & left & 287 & 24 & 3 & 89 & 60 & 10 & 4 \\
\hline German & right & 155 & 41 & 10 & 14 & 2 & 11 & 0 \\
\hline Dutch & left & 347 & 50 & 13 & 39 & 25 & 10 & 2 \\
\hline Dutch & right & 199 & 60 & 38 & 11 & 3 & 7 & 1 \\
\hline
\end{tabular}

The distribution of interfixes in left and right-branching German and Dutch compounds is summarized in Table 4 . The third column of this table again lists the 
number of left and right-branching compounds. Columns 4 and 5 show the counts of interfixes that fill the first interfixation slot (the position following the first noun), with the second interfixation slot (the position following the second noun) being empty. Column 4 lists the total number of compounds in which any interfix (-s-, -en-, $-n-,-e-,-e r-, \ldots)$ occurs after the first noun. Column 5 lists the number of such compounds in which -s- is realized, as the separation hypothesis has been advanced primarily for the -s- interfix (Grimm, 1878; Fuhrhop, 1998; 2000). Columns 6 and 7 document the counts of compounds for which only the second interfixation slot is filled. The last two columns list the counts of compounds in which both interfixation slots are filled.

The literature on German interfixation is concerned primarily with interfixation in left-branching compounds. The basic observation has been that especially the $-s$ interfix shows a preference for the second interfixation slot in left-branching compounds. Table 4 shows that in German there are indeed more interfixes in slot $2(89 / 287)$ than in slot $1(24 / 287)$ for left-branching compounds $\left(0.31\right.$ vs. $\left.0.08, \chi^{2}(1)=45.1, p<.0001\right)$, and the same observation holds for the $-s$ - interfix by itself $(3 / 287=0.01 \mathrm{vs} .60 / 287$ $\left.=0.21, \chi^{2}(1)=55.9, p<.0001\right)$. This distributional asymmetry supports the separation hypothesis, especially in the case of the $-s-$, which hardly occurs at all at the minor boundary position.

Interestingly, our data reveal not only a distributional asymmetry for leftbranching compounds, but also a similar asymmetry for right-branching compounds. Interfixes in right-branching compounds show a preference for the first slot (41/155) rather than the second slot $\left(14 / 155, \chi^{2}(1)=14.9, p=.0001\right)$, a preference that is also manifest for the $-s$ - interfix by itself $\left(10 / 155\right.$ vs. $\left.2 / 155, \chi^{2}(1)=4.2, p=.0393\right)$. This suggests that in German there is an overall asymmetry in the distribution of interfixes, with preferential occurrence in the slot of the major constituent boundary.

In Dutch, no distributional asymmetry appears to be present for left-branching compounds when we count all interfixes (50/347 is not significantly different from $\left.39 / 347, \chi^{2}(1)=1.29, p=.2563\right)$. In fact, more interfixes are realized in slot one than in slot two, contrary to what the separation hypothesis predicts. However, when we count the compounds with the $-s$ - interfix, we find a tendency in the predicted direction, although it does not reach significance $\left(13 / 347\right.$ vs. $24 / 347, \chi^{2}(1)=3.37$, $p=.06645)$. For Dutch right-branching compounds, we do see a clear distributional asymmetry, both for all interfixes counted jointly $\left(60 / 199\right.$ vs. $11 / 199, \chi^{2}(1)=39.5$, $p<.0001)$ and for the $-s$ - by itself $\left(38 / 199\right.$ vs. $\left.3 / 199, \chi^{2}(1)=31.4, p<.0001\right)$. The overall pattern suggests that in Dutch the distributional asymmetry expected under the separation hypothesis is carried primarily by the $-s$ - interfix, and that it is most clearly present in right-branching compounds.

Thus we see that the distribution of the -s-interfix in German and Dutch across the first and second interfixation slots in left and right-branching compounds reveals a clear preference for the slot coinciding with the major constituent boundary. It should nevertheless be kept in mind that the number of cases where the $-s$ - reliably marks a major constituent boundary is quite restricted. Consider the $-s$ - in German. Taking both right and left-branching compounds together, we see that $-s$ - is used in only 79 out of 442 triconstituent compounds (18\%). Nine of these 79 compounds 
contain an $-s$-at the minor constituent boundary (a noise rate of $11 \%$ ). For Dutch, we have a realization rate for the $-s$ - of $79 / 546=15 \%$ and a noise rate of $19 / 79=24 \%$.

There is a further observation to be made when we consider Tables 2 and 4 together. Table 2 shows that $2325 / 6643=35 \%$ of the biconstituent compounds in German and $3954 / 14644=27 \%$ of such compounds in Dutch contain an interfix. However, the degree of realization of interfixes in biconstituent compounds that appear as constituents in triconstituent compounds (the $\mathrm{C} 12$ compounds in leftbranching compounds and the $\mathrm{C} 23$ compounds in right-branching compounds) is much lower. Collapsing over left and right-branching compounds in German which have an interfix at the minor boundary, we count a maximum of $24+14+10+11$ $=59$ compounds. (This number represents a maximum because we have not checked whether each of the pairs of constituents surrounding the $-s$ - is in fact an existing compound of the language.) For Dutch, the corresponding count is $50+11+10+7$ $=78$. Hence, the degree of realization of interfixes in embedded compounds is at most $59 / 442=13.3 \%$ for German and $78 / 546=14.3 \%$ for Dutch. The within-language reductions in degree of realization are significant $\left(\chi^{2}(1)=86.0 ; p<.0001\right.$ for German, $\chi^{2}(1)=43.0 ; p<.0001$ for Dutch). In other words, biconstituent compounds with an interfix are relatively unproductive as base words in triconstituent compounds. If a biconstituent compound happens to already have an interfix, it apparently is less likely to be used as a constituent of a triconstituent compound. The low degree of productivity of interfixed biconstituent compounds as immediate constituents of triconstituent compounds provides further support for the separation hypothesis. Unconstrained use of interfixed biconstituent compounds would lead to, other things being equal, roughly one third of triconstituent compounds having an interfix at the minor constituent boundary, which would be detrimental to the cue-validity of the interfix as the marker of the major constituent boundary.

As mentioned above, both German and Dutch show remarkably few compounds with two interfixes. This holds both for arbitrary combinations of interfixes, as discussed above, as well as for compounds with two $-s$ - interfixes. Under chance conditions, one would expect a larger number of such compounds than is actually observed. Consider the $-s$ - interfix, the interfix for which the distributional asymmetry is most clearly present. There are four $-s-s$ - German triconstituent compounds and three in the case of Dutch (see the last column of Table 4). To gauge whether these numbers are indeed surprisingly small, we make three simple assumptions. First, we assume that in all cases of double interfixation, the interfix within the embedded compound is inherited from the lexicon, leaving one slot for further interfixation. Second, we assume that we can estimate the probability of $-s$ - interfixation by calculating the proportion of $-s$ - interfixation in biconstituent compounds. In German, this probability is .062. For Dutch, it is .145. Third, we assume that the probability of Having $\mathrm{X}$ Compounds with doubly filled slots is $(546,0.062)$-binomially distributed in the case of German, and (442, 0.145)-binomially distributed in the case of Dutch. Under these assumptions, the probabilities of having not more than four $-s-s$-compounds in German or three $-s-s$ - compounds in Dutch is extremely small $(p<.000001)$. This suggests that there is a probabilistic constraint disfavoring having two interfixes in a triconstituent compound. Interestingly, in compounds with two interfixes, the interfixes can no longer uniquely identify the main constituent boundary. Therefore, the 
scarcity of compounds with two -s- interfixes is again exactly in line with the separation hypothesis. Summing up, the scarcity of triconstituent compounds with two interfixes, the low degree of productivity of biconstituent interfixed compounds as constituents in triconstituent compounds, and the particular preference for the $-s$ - to occupy the slot coinciding with the major constituent boundary, for both left and right-branching compounds, are all consistent with the separation hypothesis.

The question now arises to what extent language users employ these distributional properties in their parsing of triconstituent compounds. What might obscure the functionality of $-s$ - interfixation is that for triconstituent compounds, the immediate constituent structure falls out naturally given that almost all triconstituent compounds consist of an existing compound and a monomorphemic noun. From this perspective, it is quite unlikely that any parsing ambiguities would arise and that an interfix would be necessary to disambiguate the immediate constituent structure. In fact, the scarcity of triconstituent compounds with an -s- interfix that might carry the separation function might be due to precisely the fact that structural ambiguities seldom arise.

In what follows, we therefore investigate experimentally whether language users nevertheless show sensitivity to the specific distributional properties of the $-s$ - interfix.

\section{Experiments}

The lexical statistics show that the interfixes, and notably so the $-s$-, reflect the hierarchical structure of triconstituent compounds. The question to be addressed now is whether the reverse might also be the case. Does the interfix codetermine how speakers of German and Dutch assign structure to triconstituent compounds?

In order to test this possibility, we make use of triconstituent compounds consisting of three nonexisting but orthographically and phonologically legal monomorphemic and monosyllabic simple words. We have opted for pseudocompounds for three reasons. The use of pseudocompounds has the advantage that we can study the role of the interfix in the absence of the great many lexical factors that codetermine the structure of existing compounds, factors such as the frequencies of the simple constituents, the frequencies of the embedded compounds, and the analogical preferences of nouns for a given interfix (or its absence). The interfix studied in our experiments is the $-s-$, as this interfix most clearly emerges from the lexical statistics as a boundary marker in both German and Dutch. The use of the $-s$ - has the additional advantage that, unlike the -en-interfix, it does not alter the syllable structure of the stimuli. We were thus able to construct, for each pseudocompound, four interfixation conditions, namely a condition with no interfix (No-S), a condition with an interfix in the left interfixation slot (Left-S), a condition with the interfix in the right slot (Right-S), and a condition with the interfix in both slots (Both-S). An example of a German pseudocompound under these four conditions is:

$\begin{array}{ll}\text { Belkfliemguhr } & \text { No-S } \\ \text { Belksfliemguhr } & \text { Left-S } \\ \text { Belkfliemsguhr } & \text { Right-S } \\ \text { Belksfliemsguhr } & \text { Both-S }\end{array}$


The last condition, with both interfixation slots filled, is one that is hardly ever realized in the language (see Table 4). We nevertheless included this condition because it provides us a means for checking that participants will not merely base their response on the first (or the last) interfix they encounter in a given compound.

Experiments 1, 2, and 4 make use of an implicit task to tap into the intuitions of speakers of German and Dutch regarding the hierarchical structure of triconstituent compounds. We asked participants to hyphenate the stimuli, the idea being that hyphenation is a natural task that does not explicitly invoke metalinguistic grammatical skills. The prediction is that participants will tend to hyphenate the compounds at the perceived major constituent boundary. In Experiment 3, we further verified these intuitions by asking participants explicitly to assign constituent structure to the stimuli.

\section{1}

\section{Experiment 1}

Materials. We constructed 60 pseudowords, each consisting of three nonexisting syllables that follow German phonotactic rules. The syllables were all constructed so that the initial and final consonant clusters were clear indicators of syllable boundaries. In this way, all pseudowords could unambiguously be interpreted as triconstituent compounds. The initial letters were capitalized as required by German spelling conventions. The coda and onset clusters of the syllables were chosen such that an -s-could only be part of the preceding syllable. Since the interfix $-s$ - is homographic with the inflectional suffix $-s$, we made sure that the phonology of the constituents ensured that the s could be interpreted as an interfix only.

Each participant saw all compounds under one of the four interfixation conditions described above. We counterbalanced the materials to guard against practice effects by grouping the items into four lists and assigning each of the four variants of a compound to a different list. The resulting four lists each contained 60 compounds, 15 compounds for each of the four conditions No-S, Left-S, Right-S, and Both-S. Each list was presented in one of three random orders, resulting in a total of 12 random orders of presentation for the experiment. Each of these lists was preceded by the same set of 10 practice items.

Procedure. Participants were told that they were going to read nonexisting threeconstituent compounds and were informed that their task in the experiment was to identify optimal hyphenation points. They were instructed to indicate the optimal hyphenation point by drawing a vertical bar between the relevant constituents. Letters were in 12 point font and were separated by three points of space to allow the vertical lines to be placed unambiguously. The experiment lasted approximately 5 mins.

Participants. Twenty-four participants, all students of the University of Vienna, volunteered to take part in the experiment. All were native speakers of German.

Results. One participant marked all compounds as left-branching. His responses were excluded from the analyses. Other participants occasionally either did not mark any boundary or marked an impossible boundary. These responses were counted as errors. Thus, the error counts group together counts of missing data and counts of 
inaccurate, sloppy boundary markings. The error rates therefore indicate how careful the participants did the experiment, and not much else. The counts of left hyphenation and right-hyphenation responses, together with the error counts, are presented in Table 5.

\section{TABLE 5}

Number of left hyphenations and right hyphenations for each of the four interfixationconditions No-S, Left-S, Right-S, and Both-S for German (Experiment 1)

\begin{tabular}{lccc} 
position of $-s-$ & left hyphenation & right hyphenation & errors \\
\hline Left-S & 176 & 161 & 8 \\
No-S & 140 & 198 & 7 \\
Right-S & 93 & 239 & 13 \\
Both-S & 116 & 217 & 12 \\
\hline
\end{tabular}

An analysis of the log odds ratio of left and right hyphenations revealed significant differences between the four hyphenation conditions, $F 1(3,66)=7.15, p=.0003$; $F 2(3,177)=14.68, p<.0001$. Participants placed more hyphenation marks after the second constituent than after the first (815 right responses, $60.8 \%$, vs. 515 left response, $\left.39.2 \%, \chi^{2}(1)=124.7, p<.0001\right)$. This is in line with the lexical statistics of German, which also show that the major constituent boundary occurs more often after the second than after the first constituent.

Pairwise comparisons of the counts of left and right responses in the four experimental conditions (with alpha $=0.05$ after Bonferroni correction, using Chi-squared tests on the summed left and right counts for each condition) revealed significant differences between all conditions except two comparisons involving the Both-S condition. This condition differed significantly from the Left-S condition, but not from the No-S condition nor from the Right-S condition. The upper left panel of Figure 1 shows the pattern of results. The vertical axis in this figure represents the proportion of right hyphenation responses on the total of nonerroneous responses.

First note that the greatest percentage of right hyphenation responses is found for the compounds in which a unique interfix follows the second constituent. Conversely, a unique interfix following the first constituent attracts left hyphenation responses. The compounds in which no interfix is present pattern in between the compounds with a unique interfix. This pattern in the experimental data matches the distributional pattern of interfixes in German.

The experiment also included a condition that is not widely used in the language, namely, compounds with two interfixes. Our lexical statistical survey revealed that there are surprisingly few such compounds. The present experiment suggests that they pattern along with both the compounds with no interfix, as well as with the compounds with an interfix in the right interfixation slot. Because the proportion of right responses for the Both-S condition does not differ significantly from the corresponding proportion for the No-S condition, one might argue that the interfixes in the Both-S condition 


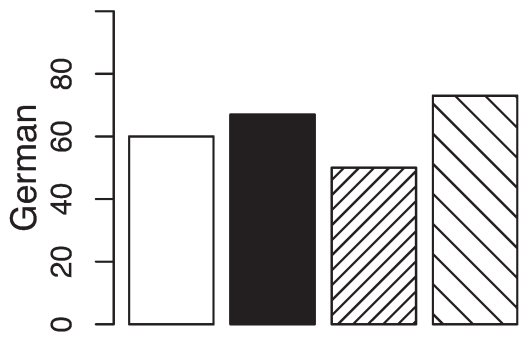

none sboth sleft sright

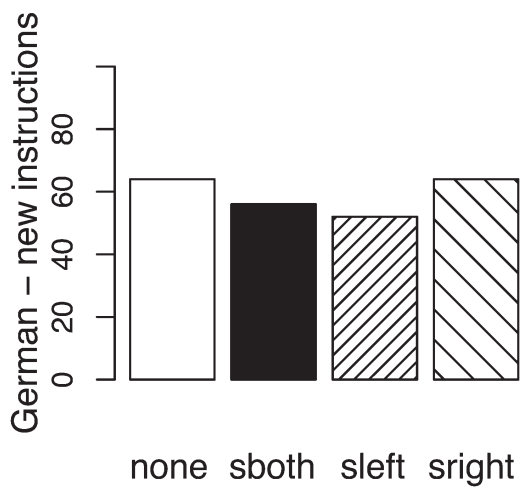

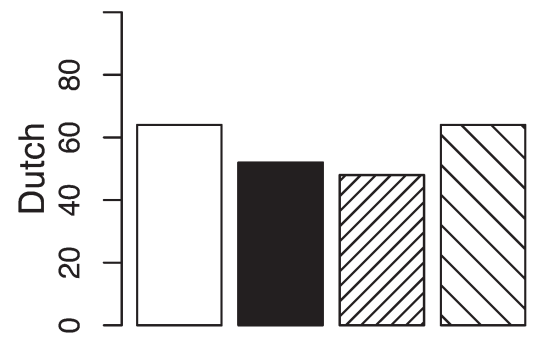

none sboth sleft sright

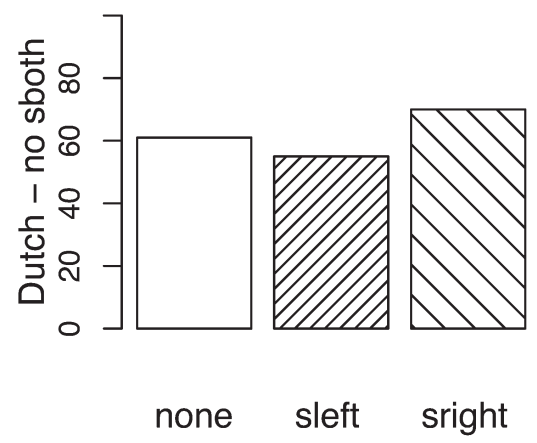

\section{Figure 1}

Percentages of right boundary responses for Experiments 1 to 4 as a function of interfixation

have no cue validity for the hierarchical structure, just as when there is no interfix at all. On the other hand, the Both-S condition also does not differ from the Right-S condition, suggesting that having the interfix in the right slot is sufficient to attract as many right responses as when there is a unique interfix in the right interfixation slot. The present data do not allow us to decide between these two interpretations.

\section{2}

\section{Experiment 2}

Materials. Similarly to the German stimuli in Experiment 1, we constructed 60 pseudowords, each consisting of three nonexisting syllables that follow Dutch phonotactics. The syllables were again of a form such that the initial and final consonant clusters were clear indicators of syllable boundaries so that each word could be unambiguously interpreted as a triconstituent compound. The coda and onset clusters of the syllables were chosen such that an -s-could only be part of the preceding syllable and not a plural suffix. As in Experiment 1, we presented each pseudocompound in 
each of the four interfixation conditions (No-S, Both-S, Left-S, Right-S), using the same counterbalancing procedure as in Experiment 1.

Procedure. The procedure was identical to that of Experiment 1.

Participants. Twenty-four participants, all students of the University of Nijmegen, were paid to take part in the experiment. All were native speakers of Dutch.

\section{TABLE 6}

Number of left hyphenations and right hyphenations for each of the four interfixation conditions No-S, Left-S, Right-S, and Both-S for Dutch (Experiment 2)

position of $-s-$

left-hyphenation

right-hyphenation

errors

Left-S

171

156

33

No-S

112

214

32

Right-S

113

214

33

Both-S

150

171

Results. Two participants marked all compounds as left-branching. Their responses were excluded from the analyses. As in Experiment 1, participants sometimes either did not mark any boundary at all or marked an impossible boundary. These responses were counted as errors and were discarded in the analyses below. There were also two missing data points, for which no response was provided. Table 6 lists the number of left and right hyphenation responses for the four conditions (No-S, Left-S, Right-S, Both-S), the upper right panel of Figure 1 shows the proportion of right hyphenation responses for each condition.

An analysis of the log odds ratio of left and right hyphenation responses revealed significant differences between the four hyphenation conditions, $F 1(3,63)=8.44$, $p<.0001 ; F 2(3,177)=11.01, p<.0001$. Participants placed more hyphenation marks after the second constituent than after the first (755 right responses, $58.0 \%$, vs. 546 left response, $42.0 \%, \chi^{2}(1)=66.5, p<.0001$ ). The higher proportion of right hyphenation responses suggests a preference for a left-branching interpretation of the compounds, a preference reflecting the predominance of left-branching compounds in Dutch.

Pairwise comparisons of the number of left and right responses in the four conditions (with alpha $=0.05$ after Bonferroni correction, using Chi-squared tests on the summed left and right counts for each condition) revealed significant differences between all conditions except two comparisons. First, the proportions of right responses did not differ significantly for the No-S and Right-S conditions. Second, the Left-S and Both-S conditions elicited very similar proportions of right hyphenation responses.

As can be seen in the upper right panel of Figure 1, the Left-S condition elicits many left hyphenation responses, while the Right-S condition elicits many righthyphenation responses. This pattern of results is very similar to that observed in Experiment 1 for German, and as in German, this pattern is what the distribution of the $-s$ - interfix in the existing compounds of Dutch leads us to expect. 
Unlike in the German experiment, the No-S condition patterns along with the Right-S condition. This would suggest that an interfix in the left interfixation slot is marked and functional, and that an interfix in the right interfixation slot is unmarked and not functional compared to compounds with no interfixes.

The Both-S condition also patterns differently than in the German experiment. In that experiment, the proportions of left and right hyphenation responses in the Both$\mathrm{S}$ condition did not differ significantly from the corresponding proportion in the Right-S condition. In the present experiment, the proportions in the Both-S and Left$\mathrm{S}$ conditions do not differ significantly. This suggests that perhaps the presence of an $-s$ - in the left interfixation slot is the crucial variable for Dutch.

These experiments assess the intuitions of speakers of German and Dutch concerning the hierarchical structure of novel compounds by means of a task that implicitly asks participants to decide on the main constituent boundary. The hyphenation task calls upon an orthographic skill and harnesses this skill to indirectly perform a metalinguistic immediate constituent analysis. In order to ensure that the results obtained do not depend on specifically this implicit task, we repeated Experiment 1, but now explicitly asked participants to parse the compounds into their immediate constituents.

\section{Experiment 3}

Materials. The materials were identical to those of Experiment 1.

Procedure. Instead of asking participants to indicate the position where they would hyphenate the word, we provided explicit parsing instructions. After informally introducing the notions of left-branching and right-branching compounds by means of two examples of existing triconstituent compounds (Steinzeitmensch, 'stone age person' and Steinfußboden, 'stone floor'), we asked them to mark the main constituent boundary.

Participants. Twenty-four participants, all students of the University of Vienna, were paid to take part in the experiment. All were native speakers of German.

Results. We excluded the responses from three participants from the analyses since they marked all compounds as left-branching. Responses with missing boundary marks or with boundary marks at improbable positions in the compound were counted as errors. Table 7 lists the number of left and right constituent boundary responses for the four conditions (No-S, Left-S, Right-S, Both-S), the lower left panel of Figure 1 shows the proportion of right hyphenation responses for each condition.

An analysis of the log odds ratio of left and right responses revealed a marginally significant difference between the four interfixation conditions for the by-subject analysis and a significant main effect for the by-item analysis, $F 1(3,60)=2.66, p=$ $.0562 ; F 2(3,177)=5.23, p<.0017$. Participants assigned more main constituent boundaries marks to the second interfixation slot than to the first slot (669 right responses, $59.7 \%$, vs. 452 left response, $40.3 \%, \chi^{2}(1)=83.2 ; p<.0001$ ), as expected.

Pairwise comparisons of the number of left and right responses in the four conditions (with alpha $=0.05$ after Bonferroni correction, using Chi-squared tests on the summed left and right counts for each condition) revealed significant differences 


\section{TABLE 7}

Number of left and right main constituent boundary responses for each of the four interfixation conditions No-S, Left-S, Right-S, and Both-S for German (Experiment 3)

position of $-s-$

left-boundary

right-boundary

errors

Left-S

142

147

26

No-S

103

192

20

Right-S

98

188

29

Both-S

115

153

only for two pairs of conditions: Left-S and Right-S, and No-S and Left-S. In other words, in this experiment, it is a unique $-s$ - in the first interfixation slot that elicited more left constituent boundary responses compared to the other conditions that are represented in the language, No-S and Right-S.

This brings us to the responses in the Both-S condition, the condition with doubly interfixed compounds that are hardly ever used in German or Dutch. Unlike in Experiment 1, in which this condition elicited fewer left hyphenation responses than the Left-S condition, no such difference is present in Experiment 3. In fact, the proportion of right responses in the Both-S condition is not statistically different from the corresponding proportion in any of the other conditions. When we consider Experiments 1-3 jointly, we find that the Both-S condition has the most variable response pattern of all conditions.

While in all experiments, one quarter of all trials contained two interfixes, the total number of compounds with two $-s$ - interfixes in our database is four for German and three for Dutch (see Table 4). The variable patterning of the responses in the Both-S condition across experiments may reflect the fact that there is no consistent and wellestablished distributional pattern in the language for participants to fall back on.

As explained above, we included the condition with two interfixes to provide us with some control for tracing task effects, allowing us to ascertain whether participants make use of some -s-spotting strategy leading them to base their response on the first or last $-s$ - encountered. However, a possible consequence of the scarcity of doubly interfixed compounds in the language and their overrepresentation in the experiments is that it may have reduced the cue validity of the interfix for the other experimental conditions. In order to ascertain to what extent the presence of doubly interfixed compounds might have biased the participants' responses, we conducted a final experiment using the same materials as in Experiment 2 but excluding the doubly marked compounds.

\section{Experiment 4}

Materials. The materials were identical of those of Experiment 2, but excluding the words in the Both-S condition. 
Procedure. The procedure was identical to that of Experiments 1 and 2.

Participants. Seventeen participants, all students of the University of Nijmegen, were paid to take part in the experiment. All were native speakers of Dutch and none of them had taken part in Experiment 2.

Results. The responses of three participants were excluded as they produced right hyphenation responses exclusively. No hyphenation responses and hyphenations at improbable positions in the compound were again counted as errors. The counts of left hyphenation and right-hyphenation responses, together with the error counts, are presented in Table 8.

\section{TABLE 8}

Number of left and right hyphenation responses for each of the three interfixation conditions No-S, Left-S, and Right-S (Dutch, Experiment 4)

position of $-s-$

left-hyphenation

right-hyphenation

errors

\begin{tabular}{lrrr} 
Left-S & 189 & 224 & 7 \\
No-S & 160 & 253 & 7 \\
Right-S & 116 & 289 & 15 \\
\hline
\end{tabular}

An analysis of the log odds ratio of left and right hyphenations revealed a significant effect of hyphenation condition, $F 1(2,26)=6.61, p=.0048 ; F 2(2,118)=13.1$, $p<.0001$. Participants also placed more hyphenation marks after the second constituent than after the first (766 right responses, $62.2 \%$, vs. 465 left response, $37.8 \%, \chi^{2}(1)$ $=146.2, p<.0001)$, as expected.

Pairwise comparisons of the counts of left and right responses in the three experimental conditions (with alpha $=0.05$ after Bonferroni correction, using Chi-squared tests on the summed left and right counts for each condition) revealed significant differences between the No-S and Right-S conditions as well as between the Left-S and Right-S conditions. The lower right panel of Figure 1 summarizes the pattern of results. The vertical axis in this figure represents the proportion of right hyphenation responses on the total of nonerroneous responses.

As in the preceding experiments, the Left-S condition elicits more left hyphenation responses while the Right-S condition elicits more right hyphenation responses. Recall that in Experiment 2, the No-S condition patterned along with the Right-S condition. We suggested that this might indicate that an interfix in the right interfixation slot might be nonfunctional. In the present experiment, omission of the Both-S condition has resulted in a different positioning of the No-S condition, which is now positioned in between the Left-S and Right-S conditions, differing significantly from the Right-S condition. In fact, the No-S condition is now no longer significantly different from the Left-S condition. Given the present experiment considered by itself, one might argue that it is precisely an interfix in the right interfixation slot which is functional. What is constant across both experiments, importantly, is the consistent difference between the Left-S and Right-S conditions. 


\section{General Discussion}

This study addressed the question of whether interfixes in German and Dutch compounds might be functional as probabilistic markers of the immediate constituent structure of multiconstituent compounds, a possibility first suggested by Grimm (1878) for German and known as the separation hypothesis.

A survey of triconstituent compounds with simplex constituents in the lexicons of German and Dutch revealed that the immediate constituent structure of compounds falls out naturally from the existing compounds that the lexicon provides as constituents. This would suggest that there would be no added functionality for interfixes. Nevertheless, it turns out that interfixes occur surprisingly often at the main constituent boundary. In German this holds for all interfixes, in Dutch, it holds only for the -s-interfix. We also observed surprisingly few triconstituent compounds with two interfixes, and that biconstituent compounds that themselves contain an interfix are significantly underrepresented as constituents of triconstituent compounds. This suggests that German and Dutch avoid interfixation at both interfixation slots in triconstituent compounds. Since an interfix can be a marker of immediate constituent structure only by virtue of there being an empty interfixation slot, we believe this provides further support for the separation hypothesis.

Having used lexical statistics to ascertain whether and how robustly main constituent boundaries attract interfixes in the existing compounds with simplex constituents of German and Dutch, we further probed the functionality of interfixation by means of a series of experiments. We investigated whether the presence of an interfix, in circumstances in which no other information could potentially guide the assignment of hierarchical structure, might prompt speakers of German and Dutch to posit a main constituent boundary at the interfixed position.

Using monosyllabic pseudowords brought together in triconstituent pseudocompounds, we observed across all experiments and tasks a significant difference in the number of posited right and left boundaries as a function of the presence of a single $-s$ - interfix. When there is a single interfix following the first constituent, this interfix gives rise to significantly more left boundary responses than when there is a single interfix following the second constituent.

The experiments investigated two further conditions, one in which no interfix was present, and one in which both interfixation slots were occupied by the $-s-$. Across experiments, we found that when the interfix is at the right interfixation slot, a number of right responses is generated that is at least as great or greater than that for the No-S condition. Conversely, when the interfix is at the left interfixation position, the number of left responses is as large as or larger than the corresponding responses for the condition with no interfix. This in-between behavior of the No-S condition is in line with the marked nature of the interfix, which according to the functional separation hypothesis provides an explicit indication of the compound's structure.

The last condition in Experiments 1-3 was one in which both interfixation slots were filled. This condition does not allow us to test the correspondence between the distributional properties of interfixes in the lexicon and the behavior of the participants, as it is a configuration that hardly ever is made use of in the language. 
Nevertheless, the condition does provide insight into the nature of the participants' performance in the experiment. If, for example, their performance was guided by positing a major boundary at the first interfix encountered, then the Both-S condition would show a predominance of left breaks, contrary to fact. Likewise, it does not reveal a systematic preference for right breaks. In fact, this condition is the most variable of all conditions in the experiment. We think this reflects the artificiality, perhaps the ungrammaticality of this condition. The contrast of this condition with the consistent pattern of responses in the two conditions with a unique interfix reinforces our confidence that the latter conditions have some real ecological validity, in that the experiments tap into the way in which the interfix in novel "grammatical" compounds guides structural interpretation.

Finally, we observed, across experiments and conditions, that there is a preference for right boundary responses. We interpret this preference as a preference for left-branching structures, the default hierarchical structure that emerged from the lexical statistics. Recall that we have restricted ourselves to compounds with simplex constituents, that is, our study is conditional on the absence of derivational affixes that may independently govern the use of particular interfixes. As the patterns of interfixation among complex words with derived constituents is not isomorphic with those observed for compounds with monomorphemic constituents (see the Appendix), we conclude that speakers of German and Dutch are sensitive to conditional probabilities of interfixation.

It is important to note that, across experiments, participants do not respond in an all-or-nothing way to the presence or absence of an interfix. The grammar of interfixation for compounds with simplex constituents is not deterministic, in which case the position of the interfix would have resulted in a nearly categorical response pattern with nearly all boundary markers following the interfix. Deterministic rules are only observed for compounds with particular derivational affixes. For compounds with simplex constituents, the presence of the interfix apparently serves as a probabilistic cue to immediate constituent structure, thanks to the nonuniform probability distribution of interfixes in these German and Dutch compounds. ${ }^{3}$

Our study therefore bears further witness to the amazing sensitivity of the human brain to subtle differences in the probability distributions of linguistic variables (see, e.g., Albright \& Hayes, 2001; Ernestus \& Baayen, in press). More than half of the triconstituent compounds with simplex constituents in German and Dutch do not contain any interfix. The distribution of the interfixes among the remaining triconstituent compounds that do have an interfix is noisy, as was documented by Table 4 . Moreover, which interfix $(-s-,-e n-,-e r-, \ldots)$ is appropriate for a given compound is itself governed by a probabilistic paradigmatic system (Krott, Baayen, \& Schreuder, 2001). In addition, compounds with derived constituents have somewhat different interfixation

3 Note that we have calculated probabilities over structured representations. Without structural information about branching direction, number of constituents, and the word category of the constituents, it would be impossible to calculate any of the distributional statistics reported in this study. We would like to emphasize that our results should not be interpreted to indicate that probability would replace structure in the grammar. 
patterns, due to the specific interfixation requirements imposed by some derivational affixes. Nevertheless, speakers of German and Dutch turn out to be sensitive to the probability distribution of the interfixes in compounds with simplex constituents, even though their functionality as markers of immediate constituent structure is almost fully redundant. This suggests to us that probability estimation is an integral part of grammatical competence, essential for the optimization of verbal communication. ${ }^{4}$

Received: April 22, 2003; revision received: December 2, 2003; accepted: December 16, 2003

\section{References}

ALBRIGHT, A., \& HAYES, B. (2001). Rules versus analogy in English past tenses: A computational/experimental study. Unpublished Manuscript.

ARONOFF, M., \& FUHRHOP, N. (2002). Restricting suffix combinations in English: Closing suffixes and the monosuffix constraint. Natural Language and Linguistic Theory, 20(3), $451-490$.

BAAYEN, R. H., PIEPENBROCK, R., \& GULIKERS, L. (1995). The CELEX lexical database (CD-ROM), Linguistic Data Consortium. Philadelphia, PA: University of Pennsylvania.

BOD, R., HAY, J. B., \& JANNEDY, S. (2002). Probability theory in linguistics. Cambridge (Mass.): The MIT Press.

BOERSMA, P., \& HAYES, B. (2001). Empirical tests of the gradual learning algorithm. Linguistic Inquiry, 32, 45-86.

BOOIJ, G. E. (2002). The morphology of Dutch. Oxford: Oxford University Press.

DRESSLER, W., KILANI-SCHOCH, M., \& KLAMPFER, S. (in press). How does a small child detect morphology. In R. H. Baayen \& R. Schreuder (Eds.), Morphological structure in language processing. Berlin: Mouton De Gruyter.

DRESSLER, W. U., LIBBEN, G., STARK, J., PONS, C., \& JAREMA, G. (2001). The processing of interfixed German compounds. In G. E. Booij \& J. van Marle (Eds.), Yearbook of morphology 1999 (pp. 185-220). Dordrecht: Kluwer.

ERNESTUS, M., \& BAAYEN, R. (in press). Predicting the unpredictable: Interpreting neutralized segments in Dutch. Language.

FLEISCHER, W. (1976). Wortbildung der deutschen Gegenwartssprache. Leipzig: Bibliographisches Institut.

4 It might be argued that speakers of German and Dutch have a deterministic rule for the selection of the interfix, with random performance noise superimposed. However, it is difficult to see how such a theory should work. Suppose that the deterministic rule follows the default, that is, no interfix. In that case, the assumption of random performance noise is incompatible with the observed preference for the interfix to appear at the major constituent boundary. Conversely, if the deterministic rule is to place the interfix at the main constituent boundary, it remains a mystery why this rule is not followed in roughly $70 \%$ of the compounds. Within the tradition of generative grammar, the stochastic optimality theory of Boersma and Hayes (2001) provides a more promising framework for understanding the present data than an account in terms of deterministic rules. Bod, Hay, and Jannedy (2002) provide a comprehensive overview of the role of probability in language and the ways in which it can be accounted for in linguistic theory. 
FUHRHOP, N. (1998). Grenzfälle Morphologischer Einheiten (Border Cases of Morphological Units). Tuebingen: Stauffenburg.

FUHRHOP, N. (2000). Zeigen Fugenelemente die Morphologisierung von Komposita an? In R. Thieroff, M. Tamrat, N. Fuhrhop, \& O. Teuber (Eds.), Deutsche Grammatik in Theorie und Praxis (pp. 201-213). Tuebingen: Max Niemeyer.

GRIMM, J. (1878). Deutsche Grammatik. Hildesheim: Olms.

HAIDER, H. (2001). Riesengratulationskompositum — *Kompositumgratulationsriesen or: Why are there no complex head-initial compounds? In C. Schaner-Wolles, J. Rennison, \& F. Neubarth (Eds.), Naturally! Linguistic studies in honor of Wolfgang Ulrich Dressler (pp. 165-174). Torino: Rosenberg \& Sellier.

KROTT, A. (2001). Analogy in morphology: The selection of linking elements in Dutch compounds. University of Nijmegen, Nijmegen.

KROTT, A., BAAYEN, R. H., \& SCHREUDER, R. (2001). Analogy in morphology: Modeling the choice of linking morphemes in Dutch. Linguistics, 39(1), 51-93.

KROTT, A., SCHREUDER, R., \& BAAYEN, R. H. (2002). A note on the function of Dutch linking elements. In G. E. Booij \& J. van Marle (Eds.), Yearbook of Morphology 2001 (pp. 237-252). Dordrecht: Kluwer.

LIBBEN, G., JAREMA, G., DRESSLER, W., STARK, J., \& PONS, C. (2002). Triangulating the effects of interfixation in the processing of German compounds. Folia Linguistica, 26, $23-43$

ORTNER, L., \& MÜLLER-BOLLHAGEN, E. (1991). Deutsche Wortbildung. Vierter Hauptteil: Deutsche Substantivkomposita. Berlin: De Gruyter.

SCHREUDER, R., NEIJT, A., Van der WEIDE, F., \& BAAYEN, R. H. (1998). Regular plurals in Dutch compounds: Linking graphemes or morphemes? Language and Cognitive Processes, 13, 551-573.

WILLIAMS, E. (1981). On the notions "lexically related" and "head of a word." Linguistic Inquiry, 12, 245-274.

ŽEPIC, S. (1970). Morphologie und Semantik der deutschen Nominalkomposita. Zagreb: Philosophische Fakultät der Universität Zagreb.

\section{Appendix}

In this study, we have restricted ourselves to triconstituent compounds with simplex constituents. The distribution of the linking elements in compounds with derived words as constituents is somewhat different, as shown in Figure 2. Each panel summarizes the proportions of compounds with simplex constituents (simplex) and the proportions of compounds with at least one derived constituent (complex) for the first and second interfixation slot, contrasting the interfix $-s$ - with the other interfixes. The left panels concern German, the right panels Dutch. The upper panels describe the left-branching compounds, and the lower panels the right-branching compounds. The counts on which these barplots are based can be found in Table 9.

Note, first of all, that compounds without any interfix are less common for the "complex" triconstituent compounds than for the "simplex" triconstituent compounds. This is a consequence of the fact that many derivational suffixes, especially in German, but also in Dutch, require a specific interfix to follow in the compound. For instance, in Dutch, a diminutive as left constituent is always followed by the $-s$-interfix. Rules such as these underlie the higher proportions of complex compounds with an $-s$ - in 
the first interfixation slot that can be observed in the upper panels of Figure 2. Further research will have to clarify how the preferences of derivational affixes for specific interfixes interact with the interfixation preferences that we have reported for compounds with monomorphemic constituents.

German, left branching compounds

Dutch, left branching compounds
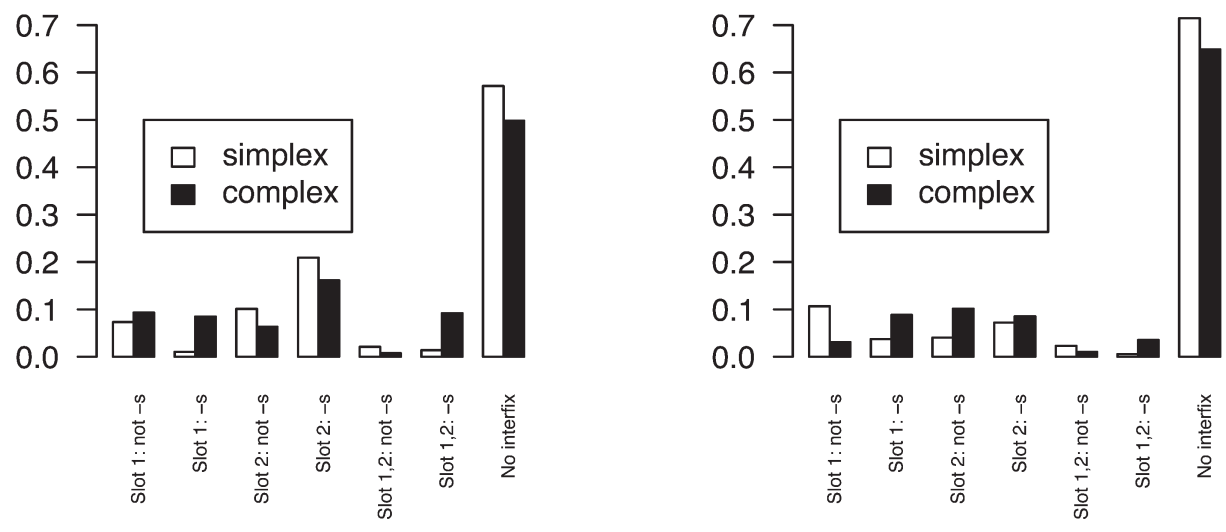

German, right branching compounds

Dutch, right branching compounds
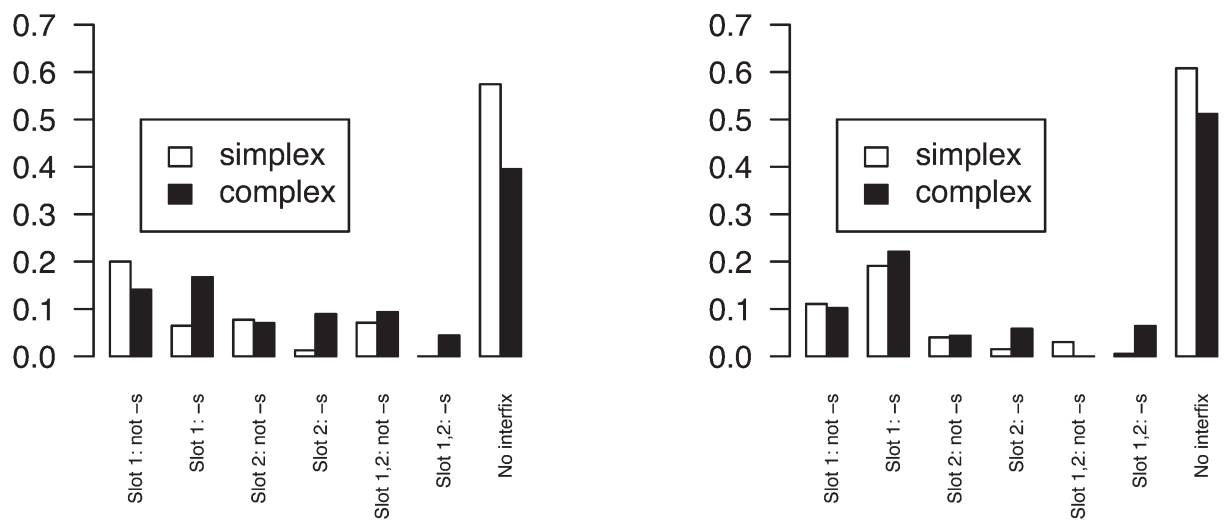

\section{Figure 2}

Distribution of interfixes across interfixation slots for left-branching and right-branching compounds in German and Dutch, broken down by morphological complexity (simplex constituents only; complex: at least one derived word as constituent 


\section{TABLE 9}

Counts of interfixes for German and Dutch triconstituent compounds, broken down by branching direction, for simplex triconstituent compounds as well as for all triconstituent compounds. The columns labeled 'not -s-' list compounds with an interfix other than -s-. The column labeled 'no interfixes' lists the counts of compounds without an interfix

\begin{tabular}{|c|c|c|c|c|c|c|c|c|c|c|}
\hline \multirow[t]{2}{*}{ branching } & \multirow[t]{2}{*}{ language } & \multirow[t]{2}{*}{ complex } & \multirow[t]{2}{*}{ total } & \multicolumn{2}{|c|}{ Slot 1} & \multicolumn{2}{|c|}{ Slot 2} & \multicolumn{2}{|c|}{ Slot 1 and 2} & \multirow[t]{2}{*}{$\begin{array}{c}n o \\
\text { interfix }\end{array}$} \\
\hline & & & & not $-s-$ & $-S-$ & not $-s-$ & $-S-$ & not $-s-$ & $-S-$ & \\
\hline left & German & simplex & 287 & 21 & 3 & 29 & 60 & 6 & 4 & 164 \\
\hline right & German & simplex & 155 & 31 & 10 & 12 & 2 & 11 & 0 & 89 \\
\hline left & German & all & 1986 & 179 & 147 & 137 & 334 & 19 & 160 & 1010 \\
\hline right & German & all & 838 & 127 & 124 & 60 & 63 & 75 & 30 & 359 \\
\hline left & Dutch & simplex & 347 & 37 & 13 & 14 & 25 & 8 & 2 & 248 \\
\hline right & Dutch & simplex & 199 & 22 & 38 & 8 & 3 & 6 & 1 & 121 \\
\hline left & Dutch & all & 1027 & 58 & 73 & 83 & 83 & 15 & 26 & 689 \\
\hline right & Dutch & all & 543 & 57 & 114 & 23 & 23 & 6 & 23 & 297 \\
\hline
\end{tabular}

